\title{
Repair of rectal perforation during robotic prostatectomy
}

\author{
Jameson Loyal, ${ }^{1}$ Roberto Bergamaschi' \\ Division of Colon and Rectal Surgery, State University of New York, Stony Brook, New York, United States of America
}

\begin{abstract}
Perforation of the rectum during robotic radical prostatectomy for prostate cancer is an uncommon but known complication. Depending on several risk factors, most convenient approach for the colorectal surgeon is to carry out a robotic hand-sewn suture repair of the perforation in 2 layers, with or without ileostomy.

Keywords: Complication; perforation; prostatectomy; rectum; repairs; robotic surgery.
\end{abstract}

\section{Introduction}

Perforation of the rectum is an uncommon but known complication occurring during radical prostatectomy for prostate cancer. ${ }^{[1]}$ In the specific case of robotic radical prostatectomy for prostate cancer,rectal perforation has been reported to occur in $0.3 \%$ to $3.8 \%$ of cases. ${ }^{[2]}$ Most rectal lacerations occur while transecting the rectourethralis muscle. Such perforations should be recognized at the time they occur in the operating room in order to minimize morbidity. When in doubt the urologist should carry out intraoperative testing for prompt detection. ${ }^{[3]}$

In our yearly volume of 250 cases of robotic radical prostatectomy for prostate cancer,the authors report two cases $(0.8 \%)$ of perforation of the anterior rectal wall, which occurred during dissection of the posterior surface of the prostate at the apex. The aim of this technical note is to describe herein our robotic approach to repair a perforation of the rectum during robotic radical prostatectomy for prostate cancer.

\section{Surgical Technique}

Once the perforation is diagnosed, the robot may remain docked in between the patient's lower limbs as per protocol for robotic prostatectomy. Before initiating the repair, the colorectal surgeon should obtain detailed information from the urologist about a number of patient-related, disease-related, and surgical technique-related factors in order to evaluate whether or not a temporary diverting loop ileostomy should follow the rectal repair. Perforation of the anterior wall of the rectum during robotic radical prostatectomy for prostate cancer can occur from sharp or thermal dissection. Although the patients may have (or not) taken a preoperative bowel preparation, a diverting ileostomy may be considered in cases of gross fecal spillage, previous radiation of the pelvis, urethrovesical anastomosis under tension, or in a patient who has been chronically treated with steroids.

The rectal repair is carried out using two robotic arms named R1 and R2. R1 is controlled by the surgeon's right 
hand operating with a needle holder. $\mathrm{R} 2$ controlled by the surgeon's left hand may use a single fenestrated grasper of various shapes according to surgeon's preference. An assistant should be available to provide suction via a laparoscopic port. The edges of the rectal wall defect should be clearly identified and repaired with interrupted sutures in two layers (Figure 1). The first layer involves absorbable, synthetic, braided polyglactin (Figure 2), whereas the second layer is carried out with silk. An air leak test should be carried out transanally after having clamped the rectum proximal to the repair with a laparoscopic atraumatic clamp with the pelvis filled of saline (Figure 3).

\section{References}

1. Häggman $M$, Brändstedt $S$, Norlen BJ. Rectal perforation after retropubic radical prostatectomy: occurrence and management. Eur Urol 1996;29:337-40.

2. Pruthi RS, Wallen EM. Robotic assisted laparoscopic radical cystoprostatectomy: operative and pathological outcomes. J Urol 2007;178:814-8.

3. Pisters LL, Wajsman Z. A simple test for the detection of intraoperative rectal injury in major urological pelvic surgery. $J$ Urol 1992;148:354.

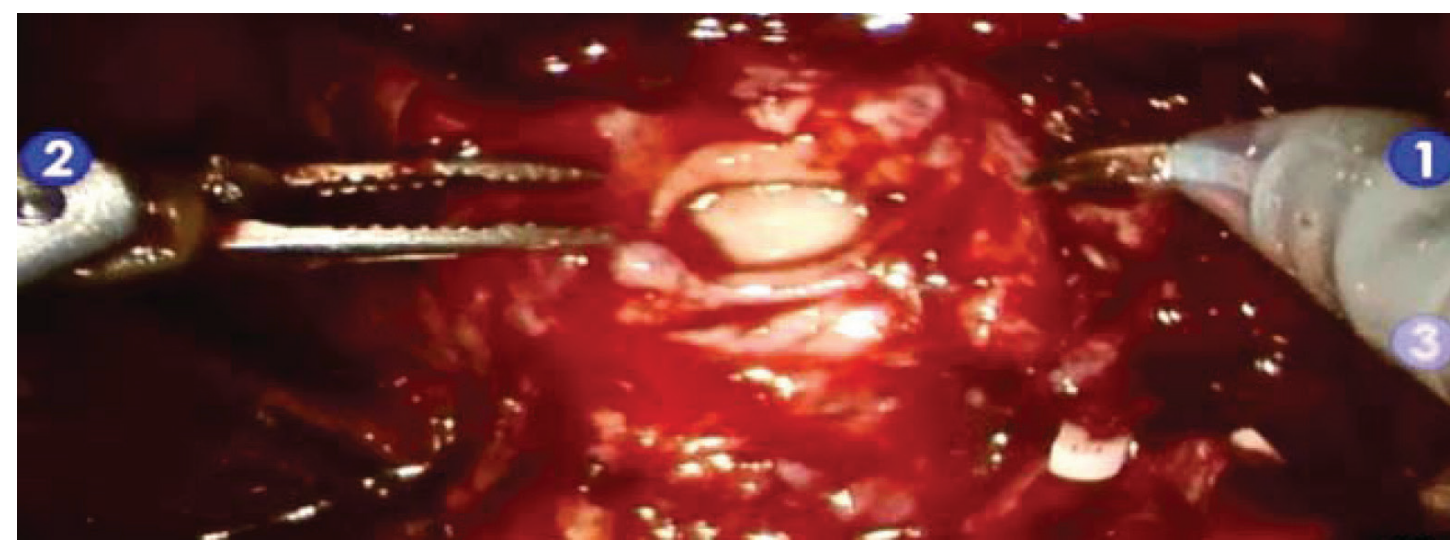

Figure 1. The edges of the rectal defect are identified.

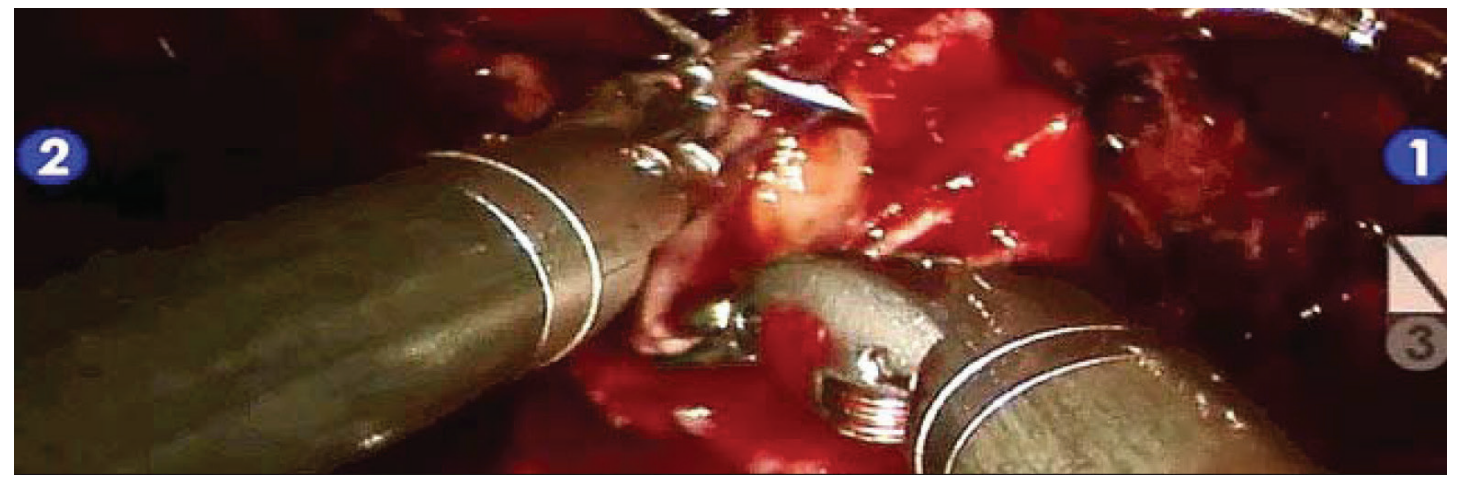

Figure 2. First layer with interrupted sutures using 3-0 polyglactin.

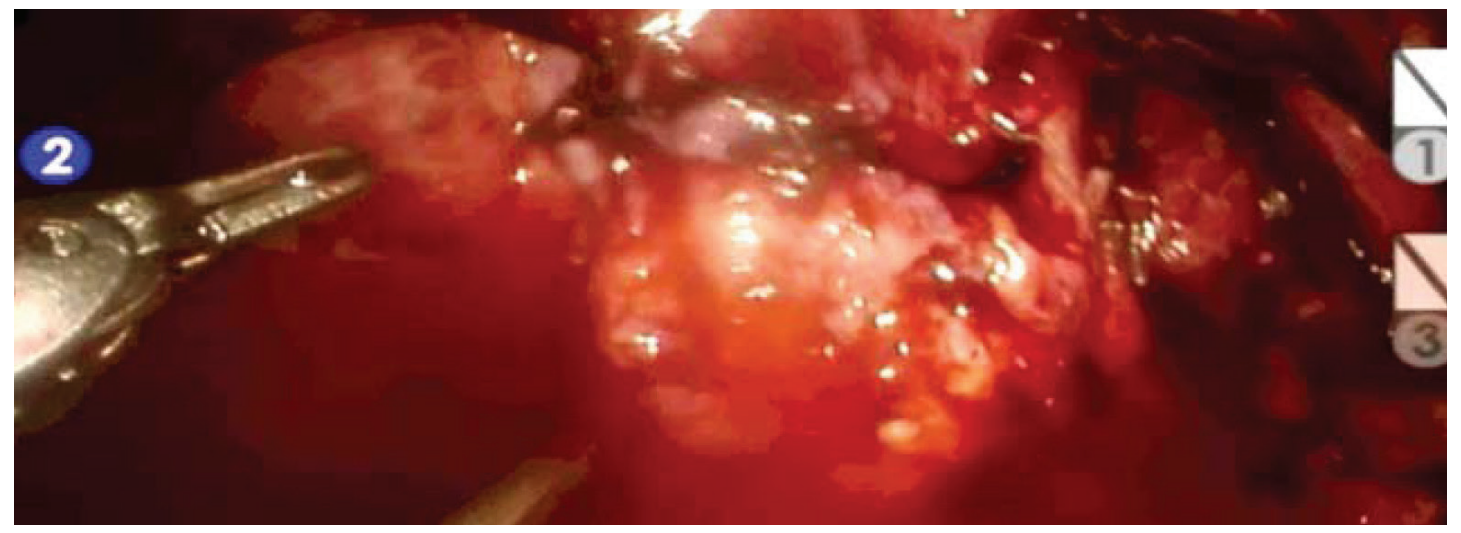

Figure 3. Air leak test with laparoscopic atraumatic clamp. 